\title{
Laparoscopic Repair of Esophageal Hiatal Hernia with Cholestasis Secondary to Pancreatic Prolapse
}

\author{
Daisuke Yagi ${ }^{11}$, Yoshikazu Takinami ${ }^{2)}$, Motoki Sugano ${ }^{1)}$ and Yasuaki Hattori ${ }^{1)}$ \\ ${ }^{1)}$ Department of Surgery, Sugita Genpaku Memorial Obama Municipal Hospital \\ ${ }^{2)}$ Department of Emergency and Critical Care Medicine, Faculty of Medicine, Shimane University
}

\begin{abstract}
The patient was an 81-year-old man who was found to have a giant hiatal hernia during detailed examination for hepatic dysfunction. Computed tomography showed prolapse of the entire stomach, head of the pancreas, and transverse colon, and part of the small bowel into the mediastinum, and magnetic resonance cholangiopancreatography showed bile duct displacement and dilatation. The patient underwent elective laparoscopic surgery for repair of the esophageal hiatal hernia. Suture closure of the hernial orifice, mesh reinforcement, and a Nissen fundoplication were performed. The postoperative clinical course has been favorable and no recurrence has occurred during the 1-year postoperative period.
\end{abstract}

Key words: esophageal hiatal hernia, cholestasis

\section{Introduction}

Esophageal hiatal hernias occur at a relatively high frequency. The stomach usually prolapses into the mediastinum, but type IV esophageal hiatal hernias exhibiting prolapse of organs other than the stomach are considered to be rare.

In this report, we describe an extremely rare case in which prolapse of the entire stomach, head of the pancreas, and duodenum caused cholestasis.

Received: April 3, 2017/ Accepted: September 26, 2017 Correspondence to: Daisuke Yagi Department of Surgery, Sugita Genpaku Memorial Obama Municipal Hospital 2-2 Otecho, Obama, Fukui 917-0078, Japan

\section{Case Report}

Patient: An 81-year-old man.

Chief complaint: None in particular.

Family history: None of note.

Medical history: Hypertension, aortic valve stenosis.

History of present illness: The patient was receiving treatment for hypertension and aortic valve stenosis as an outpatient from a local doctor, and hepatic dysfunction was observed during one such visit. Abdominal ultrasound revealed dilatation of the intrahepatic bile ducts and the patient was referred to our hospital that same day and was admitted for detailed investigation.

General examination on admission: Blood pressure was $172 / 95 \mathrm{mmHg}$, pulse 90 beats/minute, body temperature $36.7^{\circ} \mathrm{C}$, and he was anicteric. The abdomen was flat and soft with no tenderness and no rebound.

Blood tests at the time of admission: Total bilirubin (T-BIL): $1.4 \mathrm{mg} / \mathrm{dL}$; alkaline phosphatase (ALP): 1,327 IU/L; aspartate transaminase (AST): $186 \mathrm{IU} / \mathrm{L}$; alanine transaminase (ALT) $148 \mathrm{IU} / \mathrm{L}$; gamma-glutamyl transpeptidase ( $\gamma$ GTP): 175 IU/L; amylase (AMY): $68 \mathrm{IU} / \mathrm{L}$; blood urea nitrogen (BUN): $27.6 \mathrm{mg} / \mathrm{dL}$ : creatinine $(\mathrm{Cr}): 1.05 \mathrm{mg} / \mathrm{dL}$; Creactive protein (CRP): $0.23 \mathrm{mg} / \mathrm{dL}$; white blood cell (WBC) count: 4,100/ $\mu \mathrm{L}$; hemoglobin (Hb): $12.7 \mathrm{~g} /$ $\mathrm{dL}$; and platelets (PLT): $13.8 \times 10^{4} / \mu \mathrm{L}$. These findings indicated elevated hepatobiliary enzymes.

Abdominal contrast-enhanced computed tomography (CT): A severe esophageal hiatal hernia was observed, with prolapse of the entire stomach, duodenum, head of the pancreas, transverse colon, and part of the small bowel (Fig. 1). The common bile duct was occluded due to the application of cra- 

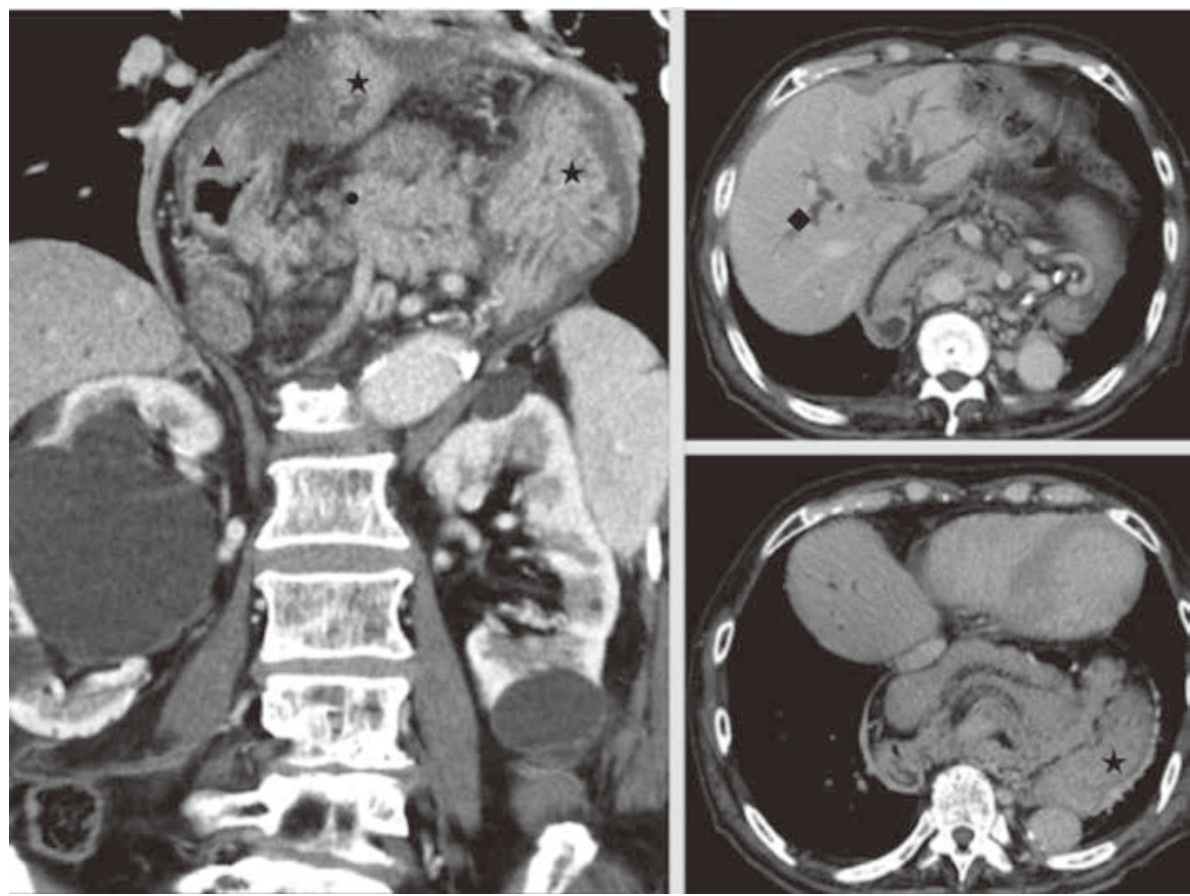

Fig. 1 Abdominal CT.

Prolapse of the entire stomach, duodenum, head of the pancreas, transverse colon, and part of the small bowel. Dilatation of the intrahepatic bile duct and common hepatic duct are observed.

$\star$ Stomach.

$\Delta$ Duodenal bulb.

OPancreas.

Intrahepatic bile duct.

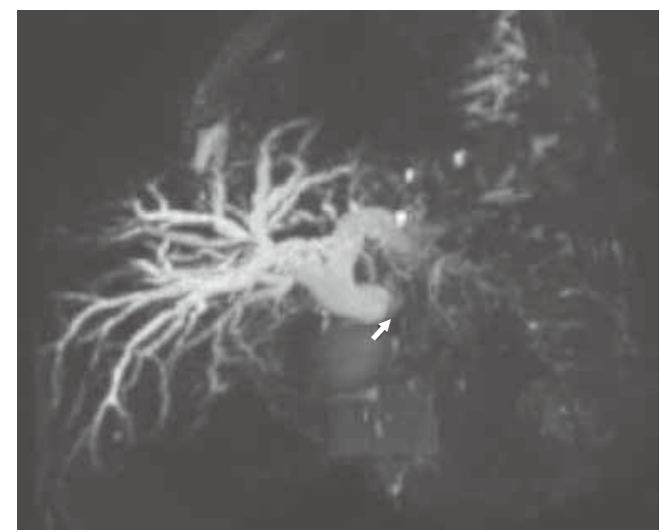

Fig. 2 MRCP.

Dilatation of the intrahepatic bile ducts, with narrowing of the common bile duct as a result of being drawn superiorly.

$\Rightarrow$ Narrowing of the common bile duct. nial traction and the intrahepatic bile duct and common hepatic duct were dilatated.

Magnetic resonance cholangiopancreatography (MRCP): Dilatation of the intrahepatic bile duct observed, but there was narrowing of the common bile duct as a result of being drawn superiorly (Fig. 2).

Upper gastrointestinal (GI) endoscopy: A giant hiatal hernia was observed with Grade C reflux esophagitis.

Based on the findings above, we made a diagnosis of a giant esophageal hiatal hernia, causing cranial traction to the common bile duct, resulting in cholestasis, which in turn caused hepatic dysfunction. The patient was diagnosed with a type IV esophageal hiatal hernia, and elective surgery was performed on hospital day 26 .

Surgical findings: Surgery was performed laparoscopically. During surgery, the patient was placed 


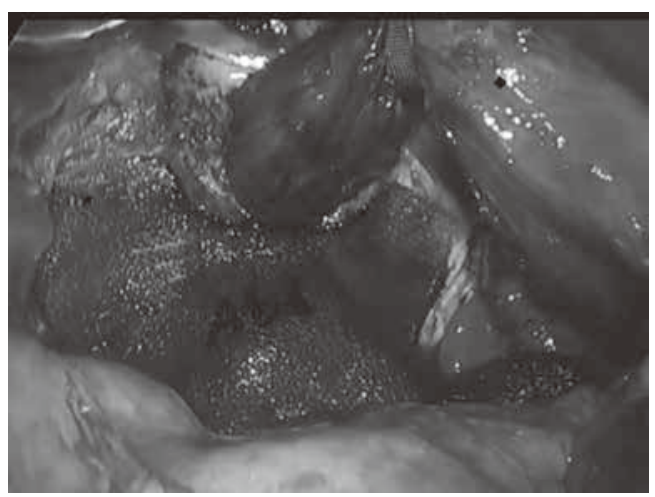

Fig. 3 Intraoperative photograph.

Mesh was used to form a concave shape to reinforce the reefed hernial orifice.

$\star$ Mesh.

$\triangle$ Esophagus.

in the supine position with the legs spread; we performed laparoscopic gastrectomy using a 5-port arrangement and a Nathanson liver retractor. The hernial orifice, which was the esophageal hiatus, was enlarged and prolapse of part of the stomach, the greater omentum, and small bowel was observed, although we were unable to confirm prolapse of the head of the pancreas. Some of the hernial contents returned into the peritoneal cavity because of positive pressure ventilation, but reduction was achieved when the omentum was returned into the abdominal cavity. The diaphragmatic crus was exposed while the stomach was drawn caudally, and the esophagus was encircled and taped while taking precautions to avoid injury to the vagus nerve. We clipped the short gastric vessels and dissected them using laparoscopic coagulating shears. The enlarged esophageal hiatus was closed by means of reefing, starting dorsally, using continuous sutures made with a $2-0$ non-absorbable barbed continuous suture thread (V-loc Wound Closure Device $^{\mathbb{R}}$; Covidien-Medtronic, Minneapolis, MN). To shorten the operative time and disperse the tension of the tissue, the diaphragm was apposed using continuous suturing, and triple knot sutures were added to reinforce the stitched area. The orifice was large, but the ventral side was arcuate. We sutured the diaphragm from the dorsal side and did not close the ventral side.

The diaphragmatic crus was sufficiently fragile to allow the opening of a pinhole, so we created a concave shape using a biodegradable coating mesh (Bard $^{\circledR}$ Ventralight ${ }^{\mathrm{TM}}$ ST; Davol Inc., Warwick, RI) and reinforced the reefed hernial orifice. We used a hernia stapler (Endo Universal ${ }^{\mathrm{TM}} 65^{\circ}$ stapler; Covidien-Medtronic) to secure the mesh and performed stapling to prevent migration of the mesh. We used staples instead of tacks to avoid complications such as pneumothorax. Paying careful attention to the vasculature of the diaphragm when using the hernia stapler, we also stapled the edges of the mesh and fixed several sites. To avoid stenosis, we also performed Nissen fundoplication after inserting an oral endoscope and secured the diaphragm and mesh using a wrap; we then ended the surgery (Fig. 3 ). The duration of surgery was $251 \mathrm{~min}$ and the total volume of blood loss was negligible (Fig. 4 ).

Postoperative clinical course: Oral intake started on postoperative day (POD) 4. There were no symptoms of reflux or stenosis and oral intake was favorable; the patient was discharged on POD 12. Blood tests 1 month postoperatively showed that the levels of hepatobiliary enzymes had all normalized. At the time of this publication, 1 year has elapsed since the surgery, but there has been no recurrence and hepatobiliary enzyme levels have remained normal. Upper GI endoscopy showed that the gastroesophageal reflux disease (GERD) has also improved.

\section{Discussion}

Esophageal hiatal hernia is a type of diaphragmatic hernia caused by increased intraabdominal pressure 


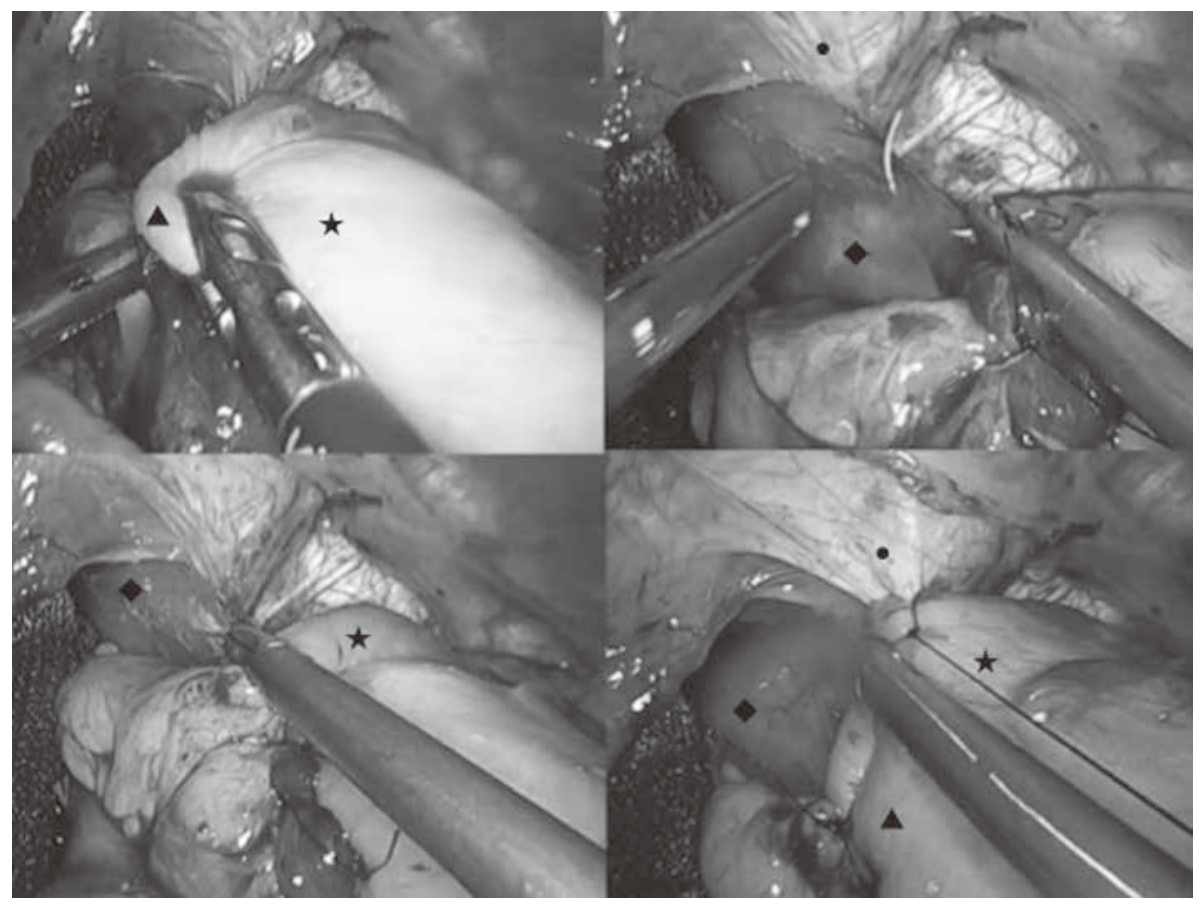

Fig. 4 Intraoperative photograph.

Nissen fundoplication after insertion of oral endoscope; the diaphragm and mesh were secured using a wrap, and the surgery was concluded.

\section{$\star$ Stomach. \\ Esophagus. \\ Diaphragm. \\ $\Delta$ Wrap.}

due to factors such as obesity, weakness of the diaphragmatic sphincter muscle associated with advancing age, and enlargement of the hiatal orifice due to age-related kyphosis. Approximately $90 \%$ of patients with this condition have the type I (sliding type), $9 \%$ have type II (mixed type), and the remaining $1 \%$ have type III (paraesophageal type) and type IV (complex type: prolapse of organs other than the stomach) $)^{1,2)}$. In an investigation of 17 cases of type IV esophageal hiatal hernia with pancreatic prolapse by Takeyama et al., the head of the pancreas prolapsed in 9 cases and caused cholestasis in only 3 cases $^{3)}$. We performed a search of "Igaku Chuo Zasshi" (ICHUSHI) for the keywords "esophageal hiatal hernia" between 1983 and 2016 and found only 3 cases of type IV esophageal hiatal hernia in which cholestasis was caused by prolapse of the head of the pancreas; our case is the fourth. Among the 3 cases reported to date, 1 case was complicated by gastric ulcer, 1 case had an upside-down stomach associated with axis rotation, and the entire stomach prolapsed into the mediastinum in all 3 cases $^{3-5)}$. In addition, in another reported case, there was no prolapse of the head of the pancreas but prolapse of the entire upside-down stomach into the mediastinum was complicated by a common bile duct stone ${ }^{6}$.

During the management of esophageal hiatal hernias, no treatment is required if a type I case is asymptomatic, but if such a case is complicated by reflux esophagitis, then proton pump inhibitors and histamine-2 receptor antagonists are administered. Surgery is performed if symptoms are poorly controlled on drug treatment or if ulceration or stenosis occurs. In type II and type III cases, the indication for surgery should be determined based on the symptoms. However, there is the potential for severe complications such as impaction, strangulation, hemorrhage, or perforation associated with enlarge- 
ment of the hernia if prolapse of organs other than the stomach occurs in type IV cases; therefore, surgical treatment is indicated as a rule ${ }^{7.8)}$. In the present case, there was dilatation of the intrahepatic bile ducts and narrowing of the common bile duct. The resulting cholestasis caused hepatic dysfunction and this was why we performed surgery.

Surgical treatment for esophageal hiatal hernia involves the following: reduction of prolapsed organ (s), exposure of both diaphragmatic crura, removal of the hernial sac and preservation of the vagus nerve, reefing of the hernial orifice, closure of the hernial orifice using a mesh, additional fundoplication, and fixation of the stomach to the diaphragm ${ }^{5}$. In 1991, Dallemagne et al. reported their initial experience with laparoscopic Nissen surgery. Since then, the number of reports of laparoscopic surgery have increased and favorable outcomes have also been reported in Japan ${ }^{9-11}$. As with other forms of laparoscopic surgery, benefits include shorter duration of postoperative hospital stay and reduced need for postoperative analgesia, although longer duration of surgery and an increased degree of surgical difficulty have been identified ${ }^{4,12}$. Opinions differ on the pros and cons of mesh use ${ }^{13-15)}$, and no definite indications have been established. However, the presence of a giant hernial orifice, such as in the present case, is a good indication for surgery because strong tension is applied during reefing and the diaphragmatic muscles are fragile. Mesh serves to prevent reexpansion of a reefed esophageal hiatus, which is usually secured such that it reinforces parts of the reefed diaphragmatic crus. The mesh that is usually used is made from either polyester and coated with a collagen film to prevent adhesions, or from polytetrafluoroethylene ${ }^{16)}$. In our case, we used Ventralight ${ }^{\mathrm{TM}}$ sculpted into a concave shape, which reinforced the diaphragmatic crus and left the ventral half of the esophageal hiatus was mesh-free.

Some reports state that fundoplication is usually unnecessary in cases without preoperative GERD ${ }^{17}$. However, reflux may occur for the first time postoperatively in cases with giant hernia, and some reports recommend that additional fundoplication should be performed in cases without preoperative reflux symptoms ${ }^{18-20)}$. Fundoplication was performed in the present case due to the presence of preoperative reflux esophagitis and the size of the giant hernia $(6.9 \times 4.8 \mathrm{~cm})$. The most common fundoplica- tion techniques are the circumferential Nissen technique and the non-circumferential Toupet technique. The Toupet method is associated with reduced incidence of postoperative dysphagia ${ }^{21)}$, although according to the GERD treatment guidelines, the Nissen method is indicated because it offers more protective effects against reflux ${ }^{22)}$. Therefore, we performed a modified Nissen fundoplication in the present $\operatorname{case}^{23)}$, which involved securing a wrap to the diaphragm and mesh using four sutures. This method was devised to prevent escape into the mediastinum. Surgery was performed in all reported cases with cholestasis, and of those, 1 was operated on laparoscopically. Reefing of the hernial orifice was performed in 2 cases with additional reinforcement using a mesh. Additional fundoplication was not performed in 2 cases, and 1 case underwent a Toupet fundoplication. Laparoscopic reefing of the hernial orifice and reinforcement using a mesh and additional Nissen fundoplication were all performed in the present case.

With regard to fundoplication, intrathoracic migration of the wrap occurs in about $7 \%$ of cases. This exerts stress on the diaphragm, resulting in features such as early postoperative vomiting and giant hernia. In addition, there have been reports of wrap breakdown, the so-called telescope phenomenon, in which the proximal part of the stomach slips through the wrap, and dysphagia occurs due to ste$\operatorname{nosis}^{24,25)}$. For gigantic esophageal hiatal hernia in which more than half of the stomach herniates, it has been reported that prolapse of the hernia gate reoccurs in $42 \%$ of cases following simple laparoscopic closure ${ }^{26}$. However, if the esophagus is too short and does not extend to within the abdominal cavity, we think that the herniated aspect of the stomach can substitute for the abdominal esophagus. An esophagus-like gastric tube can then be constructed by incising the stomach from the angle of His to the caudal side and by using a linear stapler.

We believe it is essential to consider the severity of the hernia, the presence of reflux symptoms, and patient background factors including age, body type, and medical history, and to institute flexible management on a case-by-case basis when deciding whether to use mesh, perform additional fundoplication, or use a laparoscopic approach.

In conclusion, we encountered a type IV esopha- 
geal hiatal hernia with prolapse of the head of the pancreas, which caused cholestasis. We performed laparoscopic repair and the clinical course was favorable. We believe it is important to select the surgical technique based on the pathological condition.

Conflict of interest: None.

\section{References}

1) Aoki T, Kashiwagi H, Omura N: Esophageal hiatal hernia. Clin Gastroenterol 15:757-764, 2000

2) Itano H, Okamoto $\mathrm{S}$, Kodama K, et al : Transthoracic Collis-Nissen repair for massive type IV paraesophageal hernia. Gen Thorac Cardiovasc Surg 56:446452,2008

3) Takeyama D, Ichikawa H, Ohara M, et al : Laparoscopic repair of a case of a giant esophageal hiatal hernia accompanied by impaction of the head of the pancreas causing cholestasis. J Jpn Soc Endoscopic Surg $20: 501-506,2015$

4) Monma T, Watanabe $Y$, Sakuma $T$, et al: A case of bile duct obstruction associated with an incarcerated paraesophageal hernia of the head of the pancreas. J Jpn Soc Gastroenterol Surg 43 : 229-234, 2010

5) Kumano T, Kimura Y, Komiyama S, et al : A case of mesh repair for giant hiatal hernia developed for cholangitis. Journal of Kyoto Prefectural University of Medicine 125:279-284, 2016

6) Kawachi Y, Tada T, Hatakeyama K: A case of upside-down stomach type esophageal hiatal hernia and congenital absence of the gallbladder associated with choledocholithiasis. J Jpn Pract Surgeon Soc 62 : 108-112, 2001

7) Sato S, Kitsuki H, Sumida I, et al : Hiatal herniation of the colon with digestive tract bleeding -a case report-. Jpn J Surf $20: 582-584,1990$

8) Suzuki T, Kondo S, Sato Y : Esophageal hiatal herniation of the colon accompanying sliding hernia of the stomach in an infant. Nagoya Med J 51:115119,2010

9) Martin TR, Ferguson MK, Naunheim KS : Management of giant paraesophageal hernia. Dis Esophagus $10: 47-50,1997$

10) Dallemagne B, Weerts JM, Jehaes C, et al : Laparoscopic Nissen fundoplication: preliminary report. Surg Laparosc Endosc 3:138-143, 1991

11) Kashiwagi H, Omura N, Yano F, et al : Clinical evaluation of laparoscopic fundoplication for gastroesophageal reflux disease. Jpn Soc Gastroenterol Surg $34: 345-350,2001$

12) Idani H, Asami S, Nakano K, et al : Laparoscopic surgery for a giant hiatal hernia. Operation 67 :
1391-1398, 2013

13) Koch OO, Asche KU, Berger J, et al : Influence of the size of the hiatus on the rate of reherniation after laparoscopic fundoplication and refundoplication with mesh hiatoplasty. Surg Endosc 25 : 1024-1030, 2011

14) Frantzides CT, Carlson MA, Loizides S, et al : Hiatal hernia repair with mesh: a survey of SAGES members. Surg Endosc $24: 1017-1024,2010$

15) Antonio SA, Antoniou GA, Koch OO, et al : Lower recurrence rates after mesh-reinforced versus simple hiatal hernia repair : a meta-analysis of randomized trials. Sure Laparoscopic Ends Percutan Tech $22: 498-502,2012$

16) Nomura T, Mastutani T, Hagiwara N, et al : Laparoscopic mesh repair for hiatal hernia. Nihon Ika Daigaku Igakkai Zasshi $3: 207-210,2012$

17) Morris-Stiff G, Hassn A : Laparoscopic paraesophageal hernia repair fundoplication is not usually indicated. Hernia 12:299-302, 2008

18) Patti MG, Fisichella PM : Laparoscopic paraesophageal hernia repair. How I do it. J Gastrointest Surg $13: 1728-1732,2009$

19) Furnee EJ, Draaisma WA, Gooszen HG, et al : Tailored or routine addition 20 of an antireflex fundoplication in laparoscopic large hiatal hernia repair : a comparative cohort study. World J Sung $35: 78-84,2011$

20) Lal DR, Pellegrini CA, Oelschlager BK : Laparoscopic repair of paraesophageal hernia. Surgery Clin North Am 85: 105-118, 2005

21) Broeders JA, Mauritz FA, Ahmed AU, et al : Systematic review and meta-analysis of laparoscopic Nissen (posterior total) versus Toupet (posterior partial) fundoplication for gastroesophageal reflux disease. Br J Surg 97 : 1318-1330, 2010

22) The Japanese Society of Gastroenterology eds : Gastroesophageal Reflux Disease (GERD) Treatment Guidelines. Nankodo Co., Tokyo, 2015, p93

23) Tsimoyiannis EC, Lekkas ET, Berekos K : Modified Nissen fundoplication in the treatment of gastroesophageal reflux disease. Int Surg 78:112-116, 1993

24) Soper NJ, Dunnegan D : Anatomic fundoplication failure after laparoscopic antireflux surgery. Ann Surg $229: 669-676,1999$

25) Granderath FA, Kamolz T, Schweiger UM, et al : Is laparoscopic refundoplication feasible in patients with failed primary open antireflux surgery? Surg Endosc 16 : 381-385, 2002

26) Hshemi M, Peters JH, DeMeester TR, et al : Laparoscopic repair of large type III hiatal hernia:objective followup reveals high recurrence rate. J Am Coll Surg $190: 553-560,2000$ 